\title{
Choroidal thickness, ganglion cell complex, and photoreceptor outer segment length evaluation in patients receiving tamoxifen therapy by spectral domain optical coherence tomography
}

selim bolukbasi ( $\square$ selimbolukbasi@hotmail.com )

Turkiye Cumhuriyeti Saglik Bakanligi Okmeydani Egitim ve Arastirma Hastanesi https://orcid.org/0000-0002-9957-0673

\section{Ozge Kandemir Gursel}

Turkiye Cumhuriyeti Saglik Bakanligi Okmeydani Egitim ve Arastirma Hastanesi

\section{Akin Cakir}

Turkiye Cumhuriyeti Saglik Bakanligi Okmeydani Egitim ve Arastirma Hastanesi

\section{Burak Erden}

Turkiye Cumhuriyeti Saglik Bakanligi Okmeydani Egitim ve Arastirma Hastanesi

\section{Gamze Karatas}

Turkiye Cumhuriyeti Saglik Bakanligi Okmeydani Egitim ve Arastirma Hastanesi

\section{Research article}

Keywords: tamoxifen retinopathy, choroidal thickness, ganglion cell complex, PROS, spectral-domain optical coherence tomography

Posted Date: October 12th, 2019

DOI: https://doi.org/10.21203/rs.2.10351/v2

License: (c) (i) This work is licensed under a Creative Commons Attribution 4.0 International License. Read Full License 


\section{Abstract}

Background To evaluate choroidal thickness, ganglion cell complex (GCC) and photoreceptor outer segment length were measured in patients with breast cancer undergoing tamoxifen therapy, using spectral-domain optical coherence tomography (SD-OCT); results were compared with those for normal eyes. Methods Forty-four patients with breast cancer, undergoing tamoxifen therapy, and 41 healthy controls were included in this prospective, comparative study. All participants underwent a complete ophthalmologic evaluation and SD-OCT. Subfoveal, nasal (nasal distance to fovea 500, 1000, $1500 \mu \mathrm{m}$ ), and temporal (temporal distance to fovea 500,1000, $1500 \mu \mathrm{m}$ ) choroidal thickness measurements were performed using the enhanced depth imaging mode of SD-OCT. Using an Early Treatment Diagnostic Retinopathy Study (ETDRS) circle at the macular level, the automated retinal segmentation software was applied to determine the thickness of the GCC. The photoreceptor outer segment (PROS) length was determined manually, as the distance from the inner surface of the ellipsoid zone to the inner surface of retina pigment epithelium. Results The mean choroidal thickness was statistically greater in the tamoxifen group than controls in all quadrants ( $p<0.001$ for all quadrants). Of all tamoxifen users (44 eyes of 44 patients), 33 eyes (75\%) had UCP. Pachychoroid pigment epitheliopathy (PPE) was detected in five tamoxifen-group patients (11.3\%). Patients with PPE in one eye had UCP in the fellow eye. Central serous chorioretinopathy findings were observed in one patient. Tamoxifen users had statistically lower GCC thickness in all inner rings of the ETDRS inlay and in the nasal outer ring only ( $p=0.027,0.002$, $0.002,0.001$, and 0.030 , respectively). No statistically significant difference in mean subfoveal PROS length was found between the groups. Conclusions SD-OCT provides valuable information for identifying structural changes and evaluating ocular findings in patients receiving tamoxifen therapy. Increased choroidal thickness, PPE and thinning GCC were detected in tamoxifen users. These OCT

findings may be an early indicator of retinal toxicity for patients undergoing tamoxifen therapy in the follow-up period. Keywords tamoxifen retinopathy, choroidal thickness, ganglion cell complex, PROS, spectral-domain optical coherence tomography

\section{Background}

Tamoxifen, a selective estrogen-receptor modulator, is recommended to treat early and advanced-stage breast cancer in premenopausal and postmenopausal women [1]. It reduces the available estrogen to cancer cells by competitively inhibiting the binding of estrogen to the estrogen receptors on breast tissues [2].

Tamoxifen has been used for many years, and its side effects are well documented. These include thromboembolic events, gynaecological symptoms, major depression, and musculoskeletal symptoms [3]. Ocular side effects of tamoxifen such as cataract [4], keratopathy [5], and optic neuritis [6] have been reported. Also, crystalline maculopathy [7], macular hole [8], pseudocystic foveolar cavitation [9], pacyhchoroid pigment epitheliopathy (PPE) [10], and branch retinal vein occlusion [11] have been reported as tamoxifen-associated retinal pathologies. 
We aimed to evaluate structural changes by spectral-domain optical coherence tomography (SD-OCT) before development of pathological retinal findings.

\section{Methods}

This prospective, comparative clinical study was carried out between September 2018 and January 2019 in Okmeydani Training and Research Hospital, Istanbul, Turkey. Informed consent was obtained from all participants, and the study was carried out in agreement with the Declaration of Helsinki for research involving human subjects. The first group comprised patients with breast cancer, undergoing tamoxifen therapy, following up at Okmeydani Training and Research Hospital Radiation oncology clinic; the control group comprised age- and gender-matched healthy subjects who were examined at the Eye Clinic of the same hospital. Patients who had been receiving tamoxifen therapy for less than one year were not included. All patients were receiving tamoxifen $20 \mathrm{mg}$ per a day.

Retinal diseases (such as epiretinal membrane, vein occlusion, macular hole, vitreomacular traction syndrome, or diabetic retinopathy), any systemic disorders, previous ocular surgery or trauma, congenital malformations of the eye, glaucoma and ocular hypertension, significant media opacities precluding fundus examination and/or imaging, best corrected visual acuity (BCVA) worse than 20/20, spherical equivalent refractive errors of more than \pm 3 dioptre, any medication use within the last three months, history of smoking or alcohol intake, and pregnancy were evaluated as exclusion criteria. All participants' haemoglobin, blood vitamin D, vitamin B12, iron, thyroid functional tests (T3, T4, and TSH levels), and systolic and diastolic blood pressure values were within the normal range.

A complete ophthalmic examination, including measurement of BCVA using Snellen charts, Goldmann applanation tonometry, slit-lamp biomicroscopy, and dilated fundus examination, was conducted. Axial length (AL) was evaluated using an AL-Scan optical biometer (Nidek Co., Gamagori, Japan). Choroidal thickness was measured using the enhanced depth imaging (EDI) mode of an SD-OCT (Spectralis HRA+OCT; Heidelberg Engineering Inc., Heidelberg, Germany). Twenty-five, each comprised of 40 averaged scans, were obtained in a $10^{\circ} \times 20^{\circ}$ rectangle centred on the fovea. The choroid was measured from the outer portion of the hyperreflective line corresponding to the retinal pigment epithelium-Bruch's membrane complex to the inner surface of the sclera/choroidal junction (manually drawn by the examiner). All SD-OCT measurements were performed between 10:00 and 11:00 am. Subfoveal, nasal, and temporal measurements of choroidal thickness were conducted manually. Nasal and temporal choroidal thickness measurements were carried out at $500 \mu \mathrm{m}$ intervals from the fovea centralis $(500$, 1000 and $1500 \mu \mathrm{m}$ distance to the fovea centralis; Figure 1).

Choroidal thicknesses of eyes with pachychoroid phenotype that were greater than $300 \mu \mathrm{m}$, with and without retina pigment epithelium abnormalities, were evaluated as PPE and uncomplicated pachychoroid epitheliopathy (UCP), respectively [12].

Using an Early Treatment Diagnostic Retinopathy Study (ETDRS) circle at the macular level, the automated retinal segmentation software was applied to determine thicknesses of the ganglion cell 
complex (GCC) by adding the parameters of the macular retinal nerve fibre, macular ganglion cell, and macular internal plexiform layers (Figure 2).

The photoreceptor outer segment (PROS) length was determined manually, as the distance from the ellipsoid zone to the inner surface of the retina pigment epithelium (RPE) after automatic retinal segmentation (Figure 1).

Only the right eye of each participant was evaluated for statistical analysis. Choroidal thickness and PROS length were measured manually by two independent graders without prior information about the subjects, and the averages of their measurements were used in the statistical analysis. The reliability of measurements between the two graders was evaluated using the intraclass correlation coefficient (Table 1).

Statistical analyses were performed using IBM SPSS Statistics version 21. The variables were investigated using histograms and analytical methods to test whether they are normally distributed or not. Student's $t$-test and the chi-squared test were used to compare the variables between groups. The effects of age and axial length were adjusted using ANCOVA test. A 5\% type I error level was used to infer statistical significance.

\section{Results}

A total of 85 eyes were enrolled in this prospective case-control study. Forty-four patients constituted the tamoxifen group, whereas 41 age and sex-matched subjects constituted the control group. All participants were female. Patients' mean age was $51.6 \pm 7.4$ years in the tamoxifen group and $52.0 \pm 5.5$ years in the control group $(p=0.786)$. The mean duration of tamoxifen use in the study group was $3.95 \pm$ $1.91(1-9)$ years. The groups had comparable ALs $(22.7 \pm 0.4$ vs $22.7 \pm 0.3 ; p=0.713)$.

The mean choroidal thickness was statistically greater in the tamoxifen group than the control group in all quadrants ( $p<0.001$ for all quadrants). The results are shown in Table 2. Figure 3 shows that the choroidal thickness was significantly thicker at all predefined measurement points in the tamoxifen group (all $p<0.001$ ). Among all tamoxifen users (44 eyes of 44 patients) and control group patients (41 eyes of 41 patients), 33 eyes (75\%) and 13 eyes (31.7\%), respectively, had UCP (chi-squared $=16.020 ; p<0.001$ ). PPE was detected in five patients (11.3\%) in the tamoxifen group. Patients with PPE in one eye had UCP in the fellow eye. Central serous chorioretinopathy (CSCR) findings were observed in one patient. SD-OCT and infrared reflectance (IR) images of patients with PPE and CSCR are presented in Figures 4 and 5, respectively.

Pseudocystic foveolar cavitation was observed in two patients. Fundus colour, IR, autofluorescence, fundus fluorescein angiography, and SD-OCT images of a patient with foveolar cavitation are shown in Figure 6. Crystalline retinopathy was not observed in any tamoxifen users. 
Tamoxifen users had statistically lower GCC thickness in all inner rings of the ETDRS inlay and in the nasal outer ring only $(p=0.027,0.002,0.002,0.001$, and 0.030 , respectively; Table 3$)$.

No statistically significant difference was found between the groups in mean subfoveal PROS length, mean central macular thickness (CMT), or GCC thickness in the rest of the outer rings $(p=0.640,0.144$, $0.122,0.233$, and 0.468 , respectively; Tables 4 and 3).

\section{Discussion}

The incidence of tamoxifen-associated ocular toxicity is estimated to be between $0.9 \%$ and $12 \%$ [13]. Generally, ocular toxicity occurs with high-dose therapy (cumulative dose $>100 \mathrm{~g}$ ) [14]. There are no controlled studies in the literature that evaluated structural optical coherence tomography (OCT) changes under tamoxifen therapy. Reported OCT findings associated with tamoxifen depend on case series; these findings are ellipsoid zone defect, thinning of the inner retinal layers, cystic foveal cavitation, and crystalline deposits in the inner retina [15].

In our study, the mean choroidal thickness measurements in the subfoveal, nasal, and temporal quadrants were all grater in tamoxifen users than in the control group. UCP was detected in $75 \%$ of all tamoxifen users. PPE was detected in five patients (11.3\%). The pachychoroid spectrum diseases are UCP, PPE, CSCR, pachychoroid neovasculopathy, and polypoidal choroidal vasculopathy. Characteristics of PPE are increased choroidal thickening, pathologically dilated veins in Haller's layer, thinning in Sattler's layer and the choriocapillaris layers, and variety of retina pigment epithelium abnormalities at the macula, with a lack of subretinal fluid and drusen. There is only one reported case of PPE associated with tamoxifen [10]. There are studies showing different combinations of this spectrum in the same patient $[10,16]$. In our study, the five patients with PPE in one eye had UCP in the fellow eye. We detected CSCR findings in a patient receiving tamoxifen therapy. The risk factors for CSCR are well documented; these are exogenous cortisol therapies, endogenous hypercortisolism, male sex, exogenous testosterone therapy, and polycystic ovary syndrome $[17,18,19]$. Furthermore, several studies have shown that choroidal thickness increases in various inflammatory diseases such as Vogt-Koyanagi Harada disease and Familial Mediterranean Fewer disease [20,21].

Estrogen receptors (ER) in the retina were first demonstrated in rats [22], and then both types of ER receptors (ER $a$ and ER $\beta$ ) [23] were identified in human retinas. Tamoxifen is a partial agonist, which activates ER a with lower efficacy compared to estrogen [24]. Reduced expression of ER $\mathrm{a}$ is associated with CSCR [25]. In addition, serum testosterone, cortisol, and cortisone levels increase during tamoxifen therapy [26]. Testosterone has been shown to increase choroidal vascular permeability and vascular dilatation [27]. These mechanisms may explain increasing choroidal thickness, development of PPE and CSCR in tamoxifen users.

In vitro studies have shown that tamoxifen is toxic to human RPE and photoreceptors [28]. Maculopathy associated with tamoxifen was demonstrated in previous reports. Case series in the literature include 
crystalline maculopathy [7], macular hole [8], and pseudocystic foveolar cavitation [9]. However, there are no previous reports evaluating macula, GCC thickness, and PROS length.

This study may be important in demonstrating the relationship between the use of tamoxifen and reduction in GCC thickness. Tamoxifen-associated optic neuropathy has been previously reported [6]. Retinal ganglion cell (RGC) layer thinning occurs earlier than retinal nerve fibre layer (RNFL) thinning after acute optic neuropathies, and quantification of the macular RGC layer thickness may provide more valuable information on neuronal damage than RNFL thickness does [29]. Toxic optic neuropathies are primarily due to injury of the RGC layer [30]. This suggests that ganglion cell toxicity plays an important role in the development of toxic optic neuropathy. We found that tamoxifen users had lower GCC thicknesses in all inner rings and the nasal outer ring of the ETDRS inlay. In addition to ganglion cell toxicity, the reduction in GCC thickness in tamoxifen users could be related to vascular changes at the choroidal level. Demirok et al. demonstrated that GCC is significantly reduced in both acute and chronic CSCR subjects compared with healthy subjects [31]. Increasing choroidal thickness measurements, development of PPE and CSCR, and reduced GCC thickness measurements in the tamoxifen group support this report.

There are four hyperreflective outer retinal lines that directly affect the visual prognosis on SD-OCT. These are, from inner to outer retina, the external limiting membrane, ellipsoid zone, cone outer segment tips, and RPE. Photoreceptors have two apical compartments: the inner segments and the outer segments. The PROS is located between the ellipsoid zone and RPE.

Under the fovea centralis are Müller cells and cone photoreceptors packed at their highest density, an area known as the central bouquet of cones [32]. This region is very special in terms of visual function, and disruption of architecture in this area affects visual function directly. There are some reports regarding the effect of PROS length on visual prognosis. Shiono et al. showed PROS length to be a good indicator of BCVA in the postoperative period of epiretinal membrane surgery [33]. Uslu et al. detected PROS length thinning in patients undergoing hydroxychloroquine therapy [34]. We determined that tamoxifen did not affect subfoveal PROS length.

\section{Conclusions}

There is no established monitoring to follow up ocular side effects of tamoxifen. SD-OCT may provide valuable information for understanding retinal structural changes in patients undergoing tamoxifen therapy. Even if no pathological retinal findings are detected in the fundoscopic examination, structural changes may have begun and may be detected in the SD-OCT examination. Thickening of the choroid and thinning of GCC may be early indicators of retinal toxicity for tamoxifen users in the follow-up period.

\section{Abbreviations}


AL: axial length; BCVA: best corrected visual acuity; CMT: central macular thickness; CSCR: central serous chorioretinopathy; EDI: enhanced depth imaging; ER: oestrogen receptor; ETDRS: early treatment diagnostic retinopathy study; GCC: ganglion cell complex; IR: infrared reflectance; OCT: optical coherence tomography; PPE: pachychoroid pigment epitheliopathy; PROS: photoreceptor outer segment; RGC: retinal ganglion cell; RNFL: retinal nerve fibre layer; RPE: retina pigment epithelium; SD-OCT: spectral-domain optical coherence tomography; UCP: uncomplicated pachychoroid epitheliopathy.

\section{Declarations}

\section{Acknowledgements}

Not applicable.

\section{Funding}

No financial support was received for this submission.

\section{Availability of data and materials}

The data that support the findings of this study are available from the corresponding author upon reasonable request.

\section{Authors' contributions}

SB conceived the idea. SB and OKG participated in the design of the study. OKG and GK collected the data. $A C$ and $B E$ performed the statistical analysis. $S B, O K G, A C, B E$, and $G K$ drafted and revised the manuscript. All authors were involved in manuscript preparation and further revisions. All authors read and approved the final manuscript for publication and agreed to be personally accountable for their contributions.

\section{Ethics approval and consent to participate}

All procedures performed in studies involving human participants were in accordance with the ethical standards of the institutional and/or national research committee and with the 1964 Helsinki Declaration and its later amendments or comparable ethical standards. Ethics committee approval for ophthalmological examination and to use the results of research and data analysis in breast cancer patients undergoing tamoxifen therapy was obtained from the Marmara University Medical Faculty Ethic Committee, Turkey (approval number 09.2018.427). The research adhered to the tenets of the Declaration of Helsinki. The written informed consent to participate in the study and relevant section of the manuscript was obtained from all individuals enrolled in the study.

\section{Consent for publication}

Not applicable. 


\section{Competing interests}

The authors declare that they have no competing interests.

\section{References}

1. Gnant M, Thomssen C, Harbeck N. St. Gallen/Vienna 2015: a brief summary of the consensus discussion. Breast Care 2015;10:124-30.

2. Tseng OL, Spinelli JJ, Gotay CC, Ho WY, McBride ML, Dawes MG. Aromatase inhibitors are associated with a higher fracture risk than tamoxifen: a systematic review and meta-analysis. Ther Adv Musculoskelet Dis. 2018;10(4):71-90.

3. Monnier A. Clinical management of adverse events in adjuvant therapy for hormone-responsive early breast cancer. Ann Oncol. 2007;18(8):36-44.

4. Tarafdar S, Lim LT, Collins CE, Ramaesh K. Tamoxifen keratopathy as seen with in-vivo confocal microscopy. Semin Ophthalmol. 2012;27: 27-8.

5. Paganini-Hill A, Clark LJ. Eye problems in breast cancer patients treated with tamoxifen.Breast Cancer Res Treat. 2000;60:5.

6. Gianni L, Panzini I, Li S, Gelber RD, Collins J, Holmberg SB et al. Ocular toxicity during adjuvant chemoendocrine therapy for early breast cancer: results from International Breast Cancer Study Group trials. Cancer 2006;106:505-13.

7. Srikantia N, Mukesh S, Krishnaswamy M. Crystalline maculopathy: a rare complication of tamoxifen therapy. J Cancer Res Ther. 2010;6:313-5.

8. Chung SE, Kim SW, Chung HW, Kang SW. Estrogen antagonist and development of macular hole. Korean J Ophthalmol. 2010;24:306-9.

9. Doshi RR, Fortun JA, Kim BT, Dubovy SR, Rosenfeld PJ. Pseudocystic foveal cavitation in tamoxifen retinopathy. Am J Ophthalmol. 2014;157:1291-8.

10. Ersoz MG, Arf S, Karacorlu M, Hocaoglu M, Muslubas IS. Pachychoroid pigment epitheliopathy associated with tamoxifen. Ophthalmic Surg Lasers Imaging Retina 2017;48:838-42.

11. Onder HI, Kilic AC, Kose SA, Karatas A, Kaya E, Kaya M et al. Branch retinal vein occlusion associated with tamoxifen use. Seminars in ophthalmology 2013;28:88-90.

12. Bolukbasi S, Erden B, Cakir A. Pachychoroid pigment epitheliopathy and choroidal thickness changes in coeliac disease. J Ophthalmol. 2019; DOI:10.1155/2019/6924191

13. Alwitry A, Gardner I. Tamoxifen maculopathy. Arch Ophthalmol.2002;120(10):1402.

14. Nayfield SG, Gorin MB. Tamoxifen-associated eye disease. A review. J Clin Oncol. 1996;14(3):101826.

15. Doshi RR, Fortun JA, Kim BT, Dubovy SR, Rosenfeld PJ. Pseudocysytic foveal cavitation in tamoxifen retinopathy. Am J Ophthalmol. 2014;157(6):1291-8. 
16. Dansingani KK, Balaratnasingam C, Naysan J, Freund KB. En face imaging of pachychoroid spectrum disorders with swept-source optical coherence tomography. Retina. 2016;36:499-516.

17. Liew G, Quin G, Gillies M, Fraser-Bell S. Central serous chorioretinopathy: a review of epidemiology and pathophysiology. Clin Exp Ophthalmol. 2013;41(2):201-14.

18. Nudleman E, Witmer MT, Kiss S, Williams GA, Wolfe JD. Central serous chorioretinopathy in patients receiving exogenous testosterone therapy. Retina.2014;34(10):2128-32.

19. Witmer MT, Klufas MA, Kiss S. Polycystic ovary syndrome and central serous chorioretinopathy. Ophthalmic Surg Lasers Imaging Retina. 2015;46(6):684-6.

20. Maruko I, lida T, Sugano Y, Go S, Sekiryu T. Subfoveal choroidal thickness after treatment of VogtKoyanagi-Harada disease. Retina. 2011;31:510-7.

21. Gundogan FC, Akay F, Uzun S, Ozge G, Toyran S, Genç H. Choroidal thickness changes in the acute attack period in patients with familial Mediterranean fever. Ophthalmologica. 2016;235:72-7.

22. Kobayashi K, Kobayashi H, Ueda M, Honda Y. Estrogen receptor expression in bovine and rat retinas. Invest Ophthalmol Vis Sci. 1998;39:2105-10.

23. Ogueta SB, Schwartz SD, Yamashita CK, Farber DB. Estrogen receptor in the human eye: influence of gender and age on gene expression. Invest Ophthalmol Vis Sci. 1999;40:1906-11.

24. Koulisis N, Moysidis SN, Olmos de Koo LC, Russell CA, Kashani AH. The tipping point: tamoxifen toxicity, central serous chorioretinopathy, and the role of estrogen and its receptors. Am J Ophthalmol Case Rep. 2016 May;18(3)8-13.

25. Daruich A, Matet A, Dirani A, Bousquet E, Zhao M, Farman N, et al. Central serous chorioretinopathy: recent findings and new physiopathology hypothesis. Prog Retin Eye Res. 2015;48:82-118.

26. Baumgart J, Nilsson K, Stavreus Evers A, Kunovac Kallak T, Kushnir MM, Bergquist $J$ et al. Androgen levels during adjuvant endocrine therapy in postmenopausal breast cancer patients. Climacteric. 2014;17(1):48-54.

27. Yildiz O, Seyrek M. Vasodilating mechanisms of testosterone. Exp Clin Endocrinol Diabetes. 2007;115(1):1-6.

28. Cho KS, Yoon YH, Choi JA, Lee SJ, Koh JY. Induction of autophagy cell death by tamoxifen in cultured retina pigment epithelial and photoreceptor cells. Invest Ophthalmol Vis Sci.2012;53(9):5344-53.

29. Huang-Link Y-M, Al-Hawasi A, Lindehammar H. Acute optic neuritis: retinal ganglion cell loss precedes retinal nerve fiber thinning. Neurol Sci. 2015;36:617-20.

30. Vieira LM, Silva NF, Dias dos Santos AM, dos Anjos RS, Pinto LA, Vicente AR, et al. Retinal ganglion cell analysis by optical coherence tomography in toxic and nutritional optic neuropathy. $J$ Neuroophthalmol. 2015;35(3):242-5.

31. Demirok G, Kocamaz F, Topalak Y, Altay Y, Sengun A. Macular ganglion cell complex thickness in acute and chronic central serous chorioretinopathy. Int Ophthalmol.2017;37(2):409-16. 
32. Rochon-Duvigneaud $A$. Recherches sur la fovea de la rétine humaine et particulièrement sur le bouquet des cônes centraux. Arch d'Anat Microsc . 1907;9:315-42.

33. Shiono A, Kogo J, Klose G, Takeda H, Ueno H, Tokuda N et al. Photoreceptor outer segment length: a prognostic factor for idiopathic epiretinal membrane surgery. Ophthalmology. 2013;120(4):788-94.

34. Uslu H, Gurler B, Yildirim A, Tatar MG, Kantarcı FA, Goker H, et al. Effect of hydroxychloroquine on the retinal layers: a quantitative evaluation with spectral-domain optical coherence tomography. $\mathrm{J}$ Ophthalmol. 2016; DOI:10.1155/2016/8643174

\section{Tables}

Table 1. Results for the intraclass correlation coefficient (ICC) between the two graders. ICC Confidence Interval

\begin{tabular}{l|c|cc}
\multicolumn{3}{c}{ Lower bound } & Upper bound \\
\hline Subfoveal & 0,98 & $0,96.9$ & $0,99.4$ \\
\hline Temporal 500 & 0,98 & $0,96.9$ & $0,98.7$ \\
\hline Temporal 1000 & 0,98 & $0,95,6$ & $0,98.8$ \\
\hline Temporal 1500 & 0,98 & $0,97.5$ & 0,99 \\
\hline Nasal 500 & 0.97 & $0,95.8$ & $0,98.2$ \\
\hline Nasal 1000 & 0.96 & $0,94.4$ & $0,97.7$ \\
\hline Nasal 1500 & 0.97 & $0,95.3$ & $0,98.1$ \\
\hline PROS & 0.97 & $0,95.4$ & 0,98 \\
\hline
\end{tabular}

ICC: Intraclass correlation coefficient, PROS: Photoreceptor Outer Segment

Table 2. Mean choroidal thickness measurements of tamoxifen and control groups Choroidal thickness 


\begin{tabular}{|c|c|c|c|}
\hline Tamoxifen group (n:44) & Control group (n:41) & $P$ value & \\
\hline Subfoveal (mean $\pm \mathrm{SD}, \mathrm{mm})$ & $367.9 \pm 74.2$ & $277.9 \pm 45.0$ & $<0.001$ \\
\hline (min-max) & $240-528$ & $180-388$ & \\
\hline Temporal 500 (mean \pm SD, mm) & $361.4 \pm 72.2$ & $267.9 \pm 48.8$ & $<0.001$ \\
\hline (min-max) & $245-515$ & $180-379$ & \\
\hline Temporal 1000 (mean \pm SD, mm) & $345.0 \pm 71.3$ & $262.7 \pm 49.2$ & $<0.001$ \\
\hline (min-max) & $217-515$ & $182-370$ & \\
\hline Temporal 1500 (mean \pm SD, mm) & $325.2 \pm 72.2$ & $248.4 \pm 51.5$ & $<0.001$ \\
\hline (min-max) & $209-496$ & $149-354$ & \\
\hline Nasal 500 (mean \pm SD, mm) & $350.5 \pm 74.6$ & $259.6 \pm 45.1$ & $<0.001$ \\
\hline$(\min -\max )$ & $235-514$ & $168-363$ & \\
\hline Nasal 1000 (mean士SD, mm) & $331.2 \pm 73.0$ & $247.9 \pm 44.9$ & $<0.001$ \\
\hline (min-max) & $216-510$ & $154-357$ & \\
\hline Nasal 1500 (mean士SD, mm) & $309.2 \pm 70.6$ & $233.3 \pm 49.8$ & $<0.001$ \\
\hline (min-max) & $181-482$ & $138-333$ & \\
\hline
\end{tabular}

Table 3. Mean ganglion cell complex thickness measurements in tamoxifen and control groups 


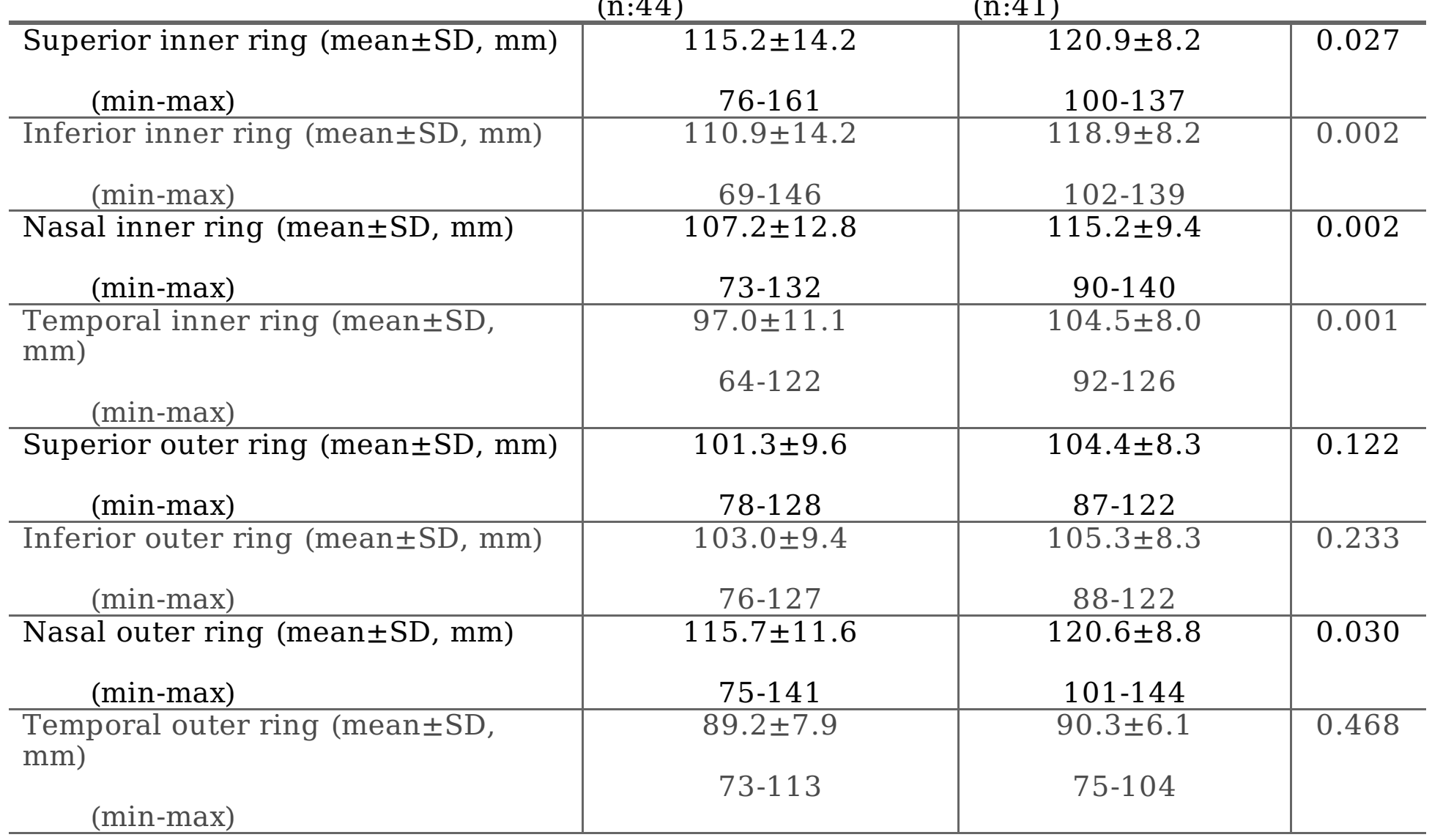

Table 4. Mean Photoreceptor Outer Segment (PROS) lenght and central macular thickness (CMT) measurements in tamoxifen and control groups

\begin{tabular}{c|c|c|c} 
& Tamoxifen group (n:44) & Control group (n:41) & P value \\
\hline Subfoveal PROS (mean \pm SD, mm) & $58.6 \pm 4.2$ & $59.0 \pm 3.8$ & 0.640 \\
(min-max) & $47-68$ & $51-69$ & \\
\hline CMT (mean \pm SD, mm) & $253.6 \pm 17.1$ & $259.4 \pm 18.7$ & 0.144 \\
(min-max) & $218-304$ & $225-299$ & \\
\hline
\end{tabular}

\section{Figures}




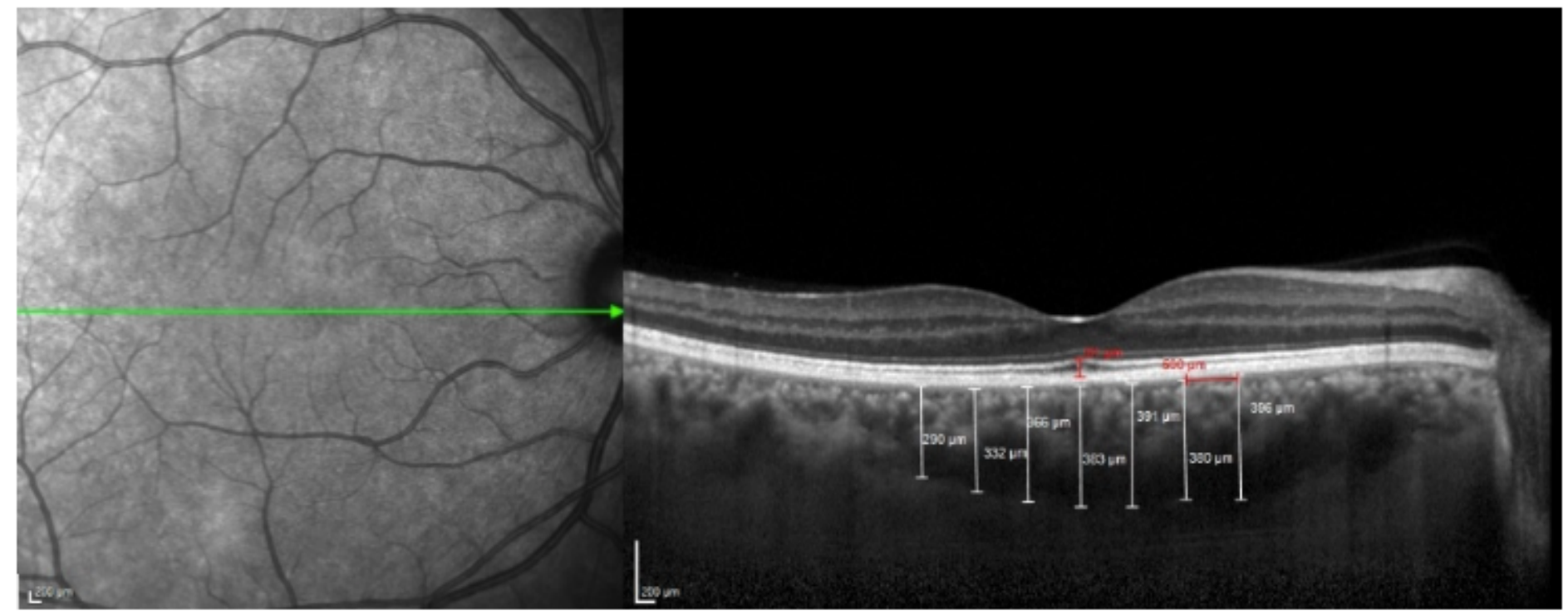

\section{Figure 1}

A representative spectral-domain optical coherence tomography (SD-OCT) image taken in enhanced depth imaging (EDI) mode showing the choroidal thickness and photoreceptor outer segment length measurements of a breast cancer patient receiving tamoxifen therapy.
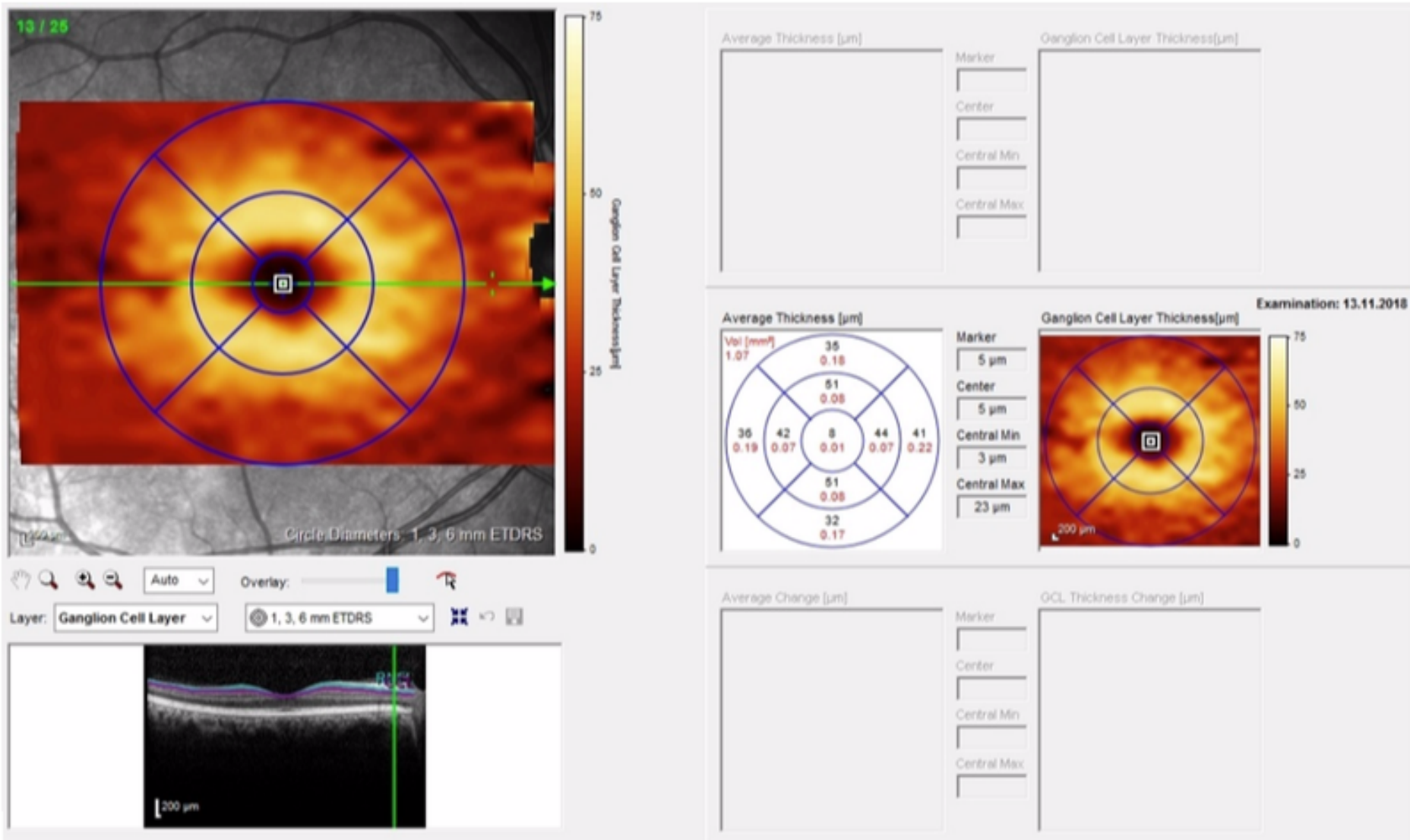

\section{Figure 2}


Retinal thickness analysis using an Early Treatment Diagnostic Retinopathy Study (ETDRS) circle at the macular level after automated retinal segmentation software was applied. In the image, the ganglion cell layer measurements of a breast cancer patient receiving tamoxifen therapy are shown as an example.

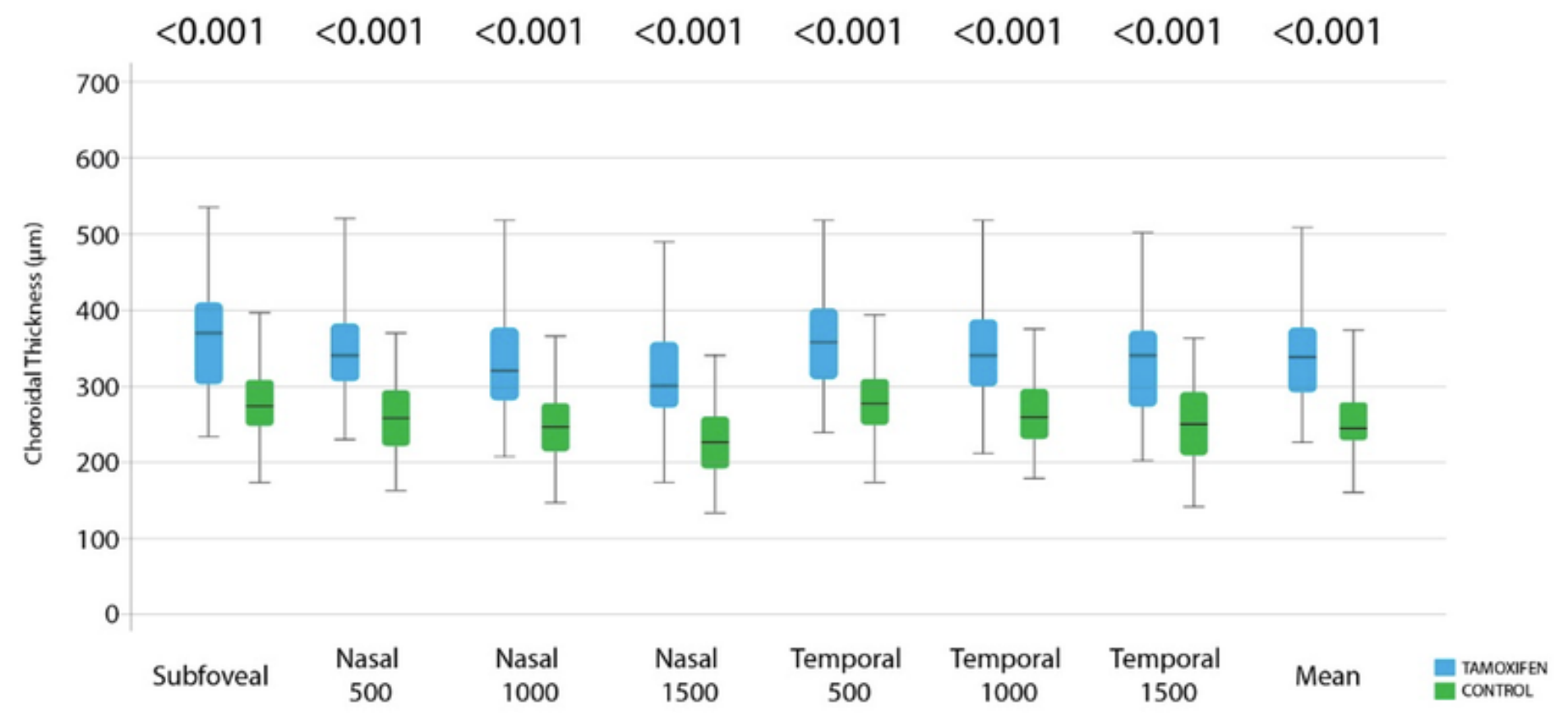

\section{Figure 3}

Comparison of choroidal thickness measurements at predefined measurement points in tamoxifen and control group. $\mathrm{P}$ values above the boxes show the statistical significance of the comparison.
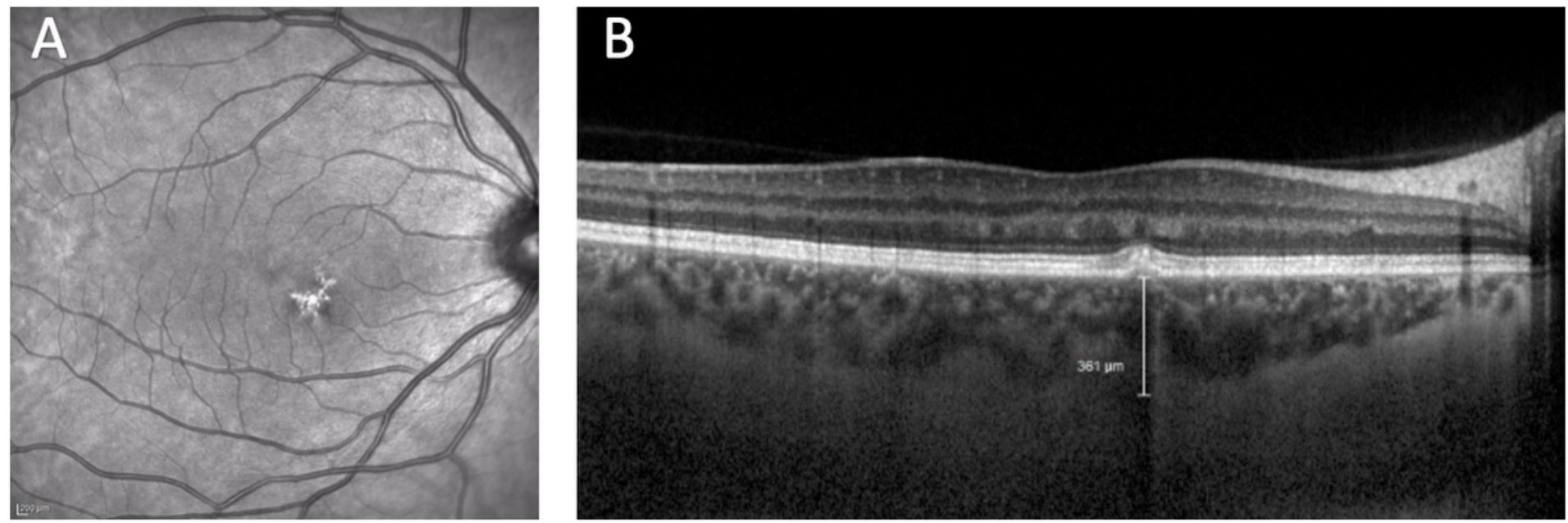

\section{Figure 4}

An infrared reflectance image (A) and a spectral-domain optical coherence tomography (SD-OCT) image taken in enhanced depth imaging (EDI) mode (B) of a 46-year-old female patient with breast cancer. She has been using tamoxifen for 5 years. Irregular hyperreflectivity appears on the infrared reflectance image of the lesion. In the SD-OCT image, pachyveins (enlargement of Haller's layer) under the retinal pigment 
epithelium (RPE) and thick choroid (choroidal thickness under RPE change: $361 \mu \mathrm{m}$ ) are seen. Subfoveal choroidal thickness is $414 \mu \mathrm{m}$.
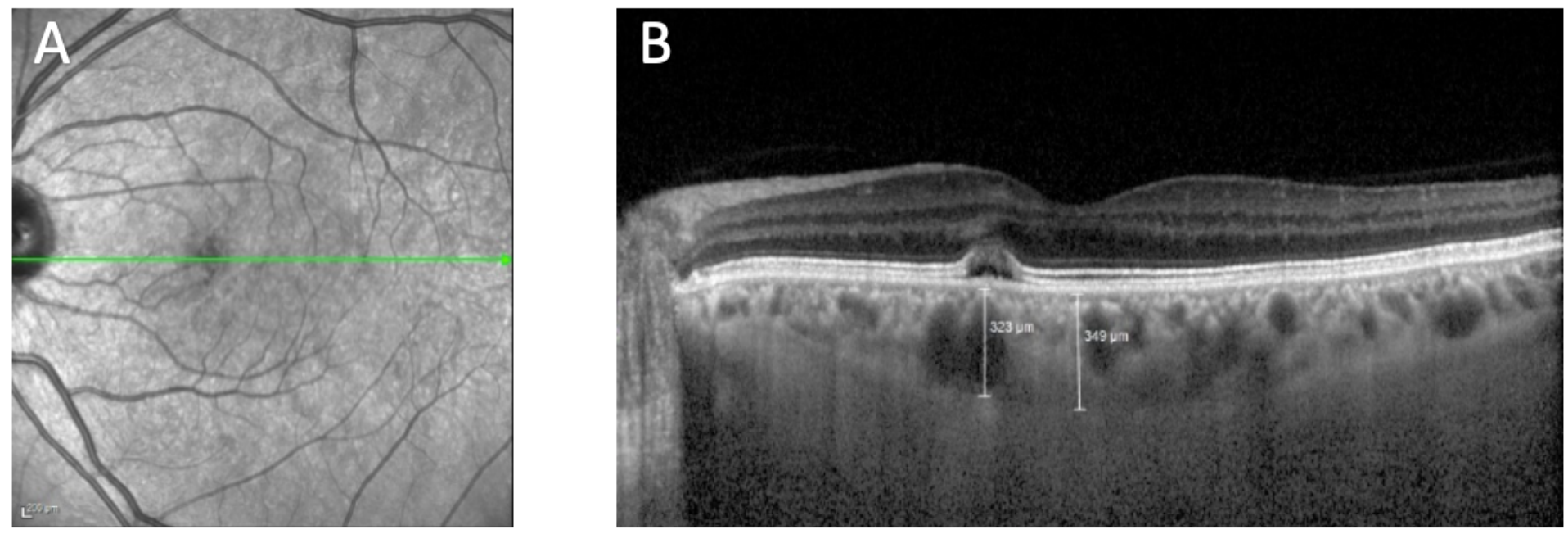

\section{Figure 5}

An infrared reflectance image (A) and a spectral-domain optical coherence tomography (SD-OCT) image taken in enhanced depth imaging (EDI) mode (B) of a 56-year-old female patient with breast cancer. She has been using tamoxifen for 7 years. A small central serous chorioretinopathy (CSCR) area is seen on the nasal side of the fovea. In the SD-OCT image, large pachyveins (enlargement of Haller's layer) under the subretinal fluid and thick choroid (choroidal thickness under CSCR: $323 \mu \mathrm{m}$ ) are seen. Subfoveal choroidal thickness is $349 \mu \mathrm{m}$. 


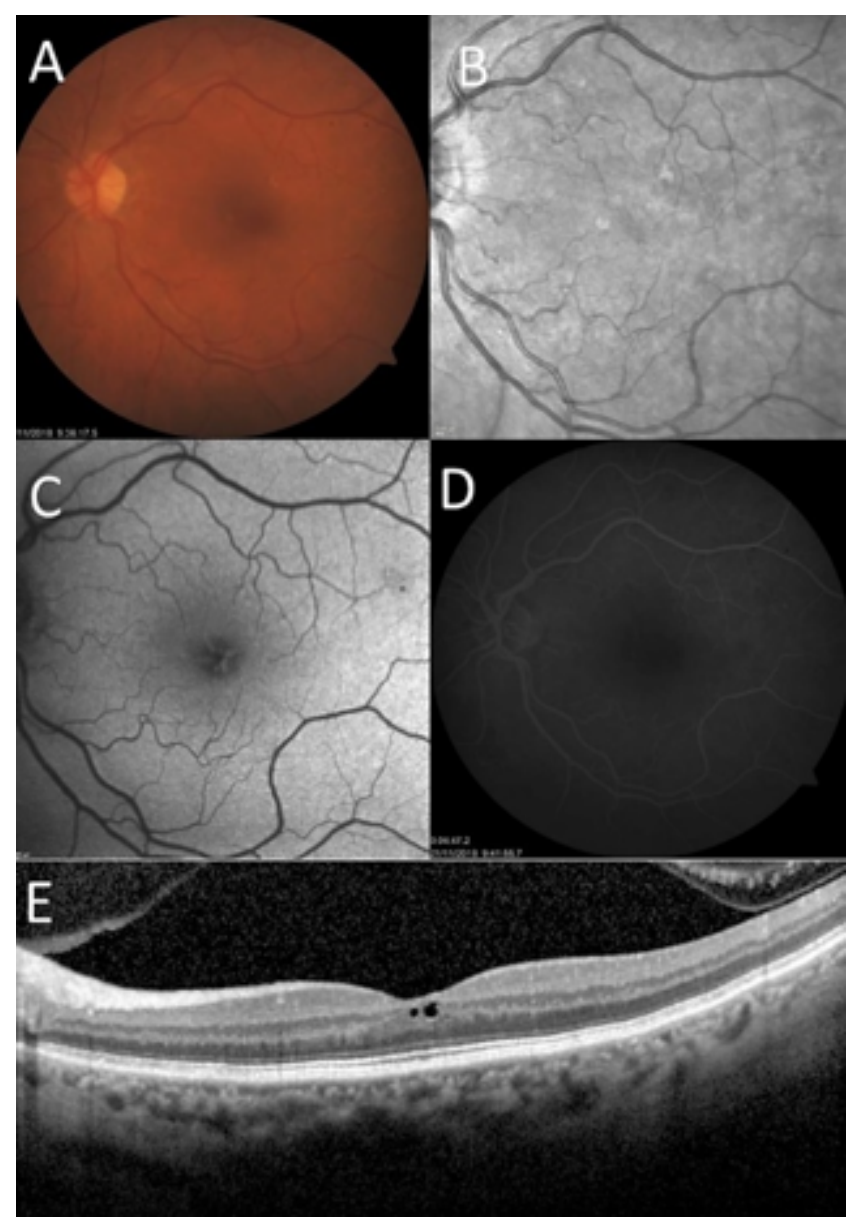

\section{Figure 6}

Left eye fundus colour, infrared reflectance (IR), fundus autofluorescence (AF), fundus fluorescein angiography (FFA), and spectral-domain optical coherence tomography images (SD-OCT) of a 58-year-old female patient with breast cancer. She has been using tamoxifen for 5 years. The IR image (B) shows normal reflectance in the foveolar region. AF (C) shows an absence of normal foveal hypoautofluorescence. SD-OCT (E) reveals foveolar cystoid spaces in the inner retina. There is no leakage on the FFA (D). 